\title{
Variabilidade de atributos do solo sob pastagens e mata atlântica na escala de microbacia hidrográfica
}

\author{
Gabriela C. Lima ${ }^{1}$, Marx L. N. Silva², Marcelo S. de Oliveira ${ }^{3}$, Nilton Curi', \\ Mayesse A. da Silva ${ }^{5}$ \& Anna H. Oliveira ${ }^{6}$ \\ ${ }^{1}$ Departamento de Ciência do Solo/UFLA. Lavras, MG. E-mail: gabscamargos@gmail.com (Autor correspondente) \\ ${ }^{2}$ Departamento de Ciência do Solo/UFLA. Lavras, MG. E-mail: marx@dcs.ufla.br \\ ${ }^{3}$ Departamento de Ciências Exatas/UFLA. Lavras, MG. E-mail: marcelo.oliveira@dex.ufla.br \\ ${ }^{4}$ Departamento de Ciência do Solo/UFLA. Lavras, MG. E-mail: niltcuri@dcs.ufla.br \\ ${ }^{5}$ Centro Internacional para Agricultura Tropical (CIAT), Colombia. E-mail: m.a.dasilva@cgiar.org \\ ${ }^{6}$ Departamento de Ciência do Solo/UFLA. Lavras, MG. E-mail: anna.ufla@gmail.com
}

\section{Palavras-chave:}

geoestatística

atributos químicos e físicos do solo

conservação do solo e da água

\begin{abstract}
R E S U M O
O solo é um dos recursos naturais de maior importância cuja heterogeneidade reflete na variabilidade de seus atributos químicos e físicos. Esta heterogeneidade é decorrente do processo de formação natural e do uso e manejo realizados pelo homem. Assim, o objetivo deste estudo foi avaliar a variabilidade espacial dos atributos químicos e físicos do solo e elaborar seus mapas de distribuição espacial na Sub-Bacia das Posses, Extrema. Para isto foram coletadas amostras em 150 pontos nas profundidades de 0-0,20 e determinados os atributos físicos e químicos. As variáveis: $\mathrm{Ca}, \mathrm{Mg}, \mathrm{K}, \mathrm{P}, \mathrm{pH}$, matéria orgânica $(\mathrm{MO})$, diâmetro médio geométrico dos agregados $\left(\mathrm{DMG}_{\mathrm{cp}}\right)$, diâmetro médio ponderado dos agregados $\left(\mathrm{DMP}_{\mathrm{cp}}\right)$ e índice de floculação (IF). IF apresentaram estrutura de dependência espacial, o que permitiu seu mapeamento a partir de técnicas geoestatísticas. O P, $D_{\mathrm{sp}}$ e $\mathrm{DMP}_{\mathrm{sp}}$ apresentaram estrutura com efeito pepita puro, possivelmente decorrente de uma distância insuficiente para captar dependência espacial na amostragem para esses atributos e interferências antrópicas. A variação verificada para o Ca pode ser explicada pela aplicação localizada de calcário, a qual foi realizada em algumas pequenas propriedades localizadas nas subbacias. O DMG $\mathrm{p}_{\mathrm{p}}, \mathrm{DMP}_{\mathrm{p}}$, e IF avaliados foram ajustados em conformidade entre si indicando boa agregação do solo.
\end{abstract}

Key words:

geostatistics

chemical and physical soil attributes

soil and water conservation

\section{Variability of soil attributes under pasturing and Atlantic Forest on the watershed basin scale}

\begin{abstract}
A B S T R A C T
Soil is one of the most important natural resource and its heterogeneity reflects the variability of its chemical and physical attributes. Soil heterogeneity occurs due to the natural process of formation and use by men and management. The objectives of this study were to evaluate the spatial variability of physical and chemical soil attributes and map the spatial distribution of these in Posses sub-basin, Extrema, representative of the Atlantic Forest biome present in southern Minas Gerais. For this, samples (0-0.20 m depth) were collected at 150 points, and their physical and chemical attributes were determined. Variables: $\mathrm{Ca}, \mathrm{Mg}, \mathrm{K}, \mathrm{P}, \mathrm{pH}$, organic matter (MO), geometric mean diameter of aggregates $\left(\mathrm{DMG}_{\mathrm{cp}}\right.$ ) weighted mean diameter of aggregates $\left(\mathrm{DMP}_{\mathrm{cp}}\right)$ and flocculation index (IF) showed spatial dependence structure, which allowed mapping from geostatistical techniques. $\mathrm{P}, \mathrm{DMG}_{\mathrm{sp}}$ and $\mathrm{DMP}_{\mathrm{sp}}$ presented structure with nugget effect, possibly due to an insufficient distance to capture spatial dependence in the sample for these attributes, and human interferences. The variation for Ca can be explained due to lime application, which occurred in some small properties located in the sub-basin. The $\mathrm{DMG}_{\mathrm{cp}}, \mathrm{DMP}_{\mathrm{cp}}$, and IF evaluated were adjusted in line with each other, indicating good soil aggregation.
\end{abstract}

\section{INTRODUÇÃO}

O Brasil é um país rico em recursos naturais distribuídos em seus diversos biomas. A Mata Atlântica é considerada, atualmente, seu bioma mais importante em razão do seu estado crítico - nela se concentram cerca de $70 \%$ da população brasileira e o percentual de remanescentes bem conservados, é de apenas $7,26 \%$. Apesar da devastação acentuada a Mata Atlântica ainda é responsável por garantir o abastecimento de água de mais de 120 milhões de brasileiros. Seus rios e córregos constituem importantes bacias e sub-bacias hidrográficas responsáveis por regular o fluxo de importantes mananciais hídricos do Brasil (Ribeiro et al., 2009).

A Mata Atlântica e os demais biomas proporcionam, em suas condições naturais de equilíbrio, de forma gratuita e contínua, uma série de benefícios ao homem, nos quais se incluem a regulação climática, ciclagem de nutrientes e a manutenção das condições dos recursos ambientais naturais, em especial 
a biodiversidade e a variabilidade genética, de que o homem retira elementos essenciais para a melhoria da produção animal e vegetal.

As consequências advindas da perda desses recursos oferecidos pela natureza têm sido, muitas vezes, catastróficas. Estudos relacionados ao desastre ocorrido na região serrana do Rio de Janeiro em janeiro de 2011, constataram que as maiores perdas e destruições ocorreram em áreas consideradas, por lei, como de preservação permanente, as quais estavam ocupadas inadequadamente por uso antrópico (MMA, 2011).

Na Sub-Bacia das Posses, localizada no município de Extrema, sul do estado de Minas Gerais, a ocupação antrópica em área de preservação permanente vem comprometendo os remanescentes de Mata Atlântica na região, danificando o solo, a água e as florestas.

Hoje, a ocupação antrópica na sub-bacia é bem visível. A presente área é ocupada, em quase sua totalidade (70\%) por gramíneas do tipo braquiária sendo alguns pontos caracterizados como pastagem degradada (Lima, 2010). A Sub-Bacia das Posses se encontra inserida no Bioma da Mata Atlântica fazendo parte do Sistema Cantareira, o maior sistema produtor de água do mundo. A disponibilidade de água na região pode ser afetada pela condição degradada dos atributos solo, cobertura vegetal e topografia da região.

Uma técnica que auxilia a avaliação e a compreensão dos recursos naturais é a geoestatística, que permite descrever a continuidade espacial, característica essencial de muitos fenômenos naturais. A geoestatística oferece um conjunto de ferramentas estatísticas que incorporam, no processamento dos dados, as coordenadas espaciais das observações (Isaaks \& Srivastava, 1989).

O interesse da análise geoestatística não se limita à obtenção de um modelo de semivariograma e seus parâmetros, desejandose também predizer valores em pontos não amostrados. Neste segmento Souza et al. (2010) avaliaram o comportamento de atributos químicos do solo interpolando pontos amostrados e não amostrados, em Alegre, ES.

Estudos desenvolvidos a partir da espacialização de atributos químicos e físicos do solo sinalizaram que a geoestatística foi uma ferramenta viável para identificar áreas de inconsistência em termos de correção da acidez do solo e definir melhores dosagens e tipos de calcário para diferentes zonas de acidez (Hurtado et al., 2010).

A avaliação da variabilidade espacial dos atributos do solo vem sendo utilizada através de ferramentas da geoestatística como fonte eficiente para o conhecimento da relação das propriedades do solo no espaço (Grego \& Vieira, 2005). Sendo adequada no estudo de um manejo que vise aperfeiçoar as práticas culturais na produção e a conservação do solo (Cambardella et al., 1994).

Assim, o objetivo deste estudo foi avaliar a variabilidade espacial dos atributos químicos e físicos do solo e elaborar mapas de distribuição espacial utilizando técnicas estatísticas descritivas e procedimentos geoestatísticos na análise dos dados, para subsidiar a identificação das áreas com maior suscetibilidade à erosão hídrica, na Sub-Bacia Hidrográfica das Posses, Extrema, MG, representativa do Bioma Mata Atlântica.

\section{Material e Métodos}

A área de estudo possui $1.196,70$ ha $\left(11,97 \mathrm{~km}^{2}\right)$ e compreende a Sub-Bacia Hidrográfica das Posses, localizada no município de Extrema, no sul do Estado de Minas Gerais. Esta Sub-Bacia está inserida na Bacia Hidrográfica do Rio Jaguari, um dos rios que abastecem o Reservatório do Sistema Cantareira no Estado de São Paulo e se encontra dentro do bioma da Mata Atlântica (ANA, 2008).

Situa-se entre as coordenadas UTM 374.500 e 371.500 de longitude E e entre 7.468.200 e 7.474.800 de latitude S (Datum SAD 69, Zona 23S) e entre as altitudes de 1.144 e $1.739 \mathrm{~m}$ (bacia de cabeceira). O clima na Sub-Bacia é do tipo Cwb, de acordo com a classificação de Köppen. A temperatura média anual é de $18{ }^{\circ} \mathrm{C}$ tendo, nos meses mais quentes e mais frios, temperaturas médias de 25,6 e $13,1^{\circ} \mathrm{C}$, respectivamente, com ocorrência de geadas anuais e precipitação média anual de 1.477 mm (ANA, 2008).

As classes de solo predominantes na Sub-Bacia são: Cambissolo Háplico, ocupando 3,52 $\mathrm{km}^{2}$ (29\% da área) o Cambissolo Húmico, $1,09 \mathrm{~km}^{2}$ (10\%), o Argissolo VermelhoAmarelo 4,79 km² (40\%), o Neossolo Flúvico 1,09 km² (10\%) e o Neossolo Litólico 1,36 km² (11\%) (Figura 1A). As fases de relevo predominantes são ondulado e forte ondulado (Figura 1B). Atualmente, o principal uso do solo é pastagem (Lima, 2010) sendo que grande parte se encontra mal-manejada; há, também, povoamentos de eucalipto, culturas anuais e mata nativa (Figura $1 \mathrm{C}$ ).

Anterior à coleta das amostras foi gerada uma malha regular de amostragem do solo contendo 150 pontos espaçados em até $350 \mathrm{~m}$ e distribuídos em uma área de aproximadamente 2 ha (Figura 2). É importante salientar que a malha dita "regular", não foi gerada com pontos equidistantes entre si, como deveria, devido a problemas de locação. Alguns pontos eram inacessíveis por se situarem em elevadas altitudes e matas densas, por exemplo. Além disto, objetivou-se coletar amostras de maneira representativa para as cinco classes de solos predominantes na Sub-Bacia e principais usos.

Para o processo de marcação dos pontos foi utilizado o sistema de posicionamento global (GPS). A marcação das coordenadas em cada ponto foi realizada pelo aparelho de GPS GARMIN e Trex Vista. O solo foi coletado em cada ponto de amostragem na profundidade de $0-0,20 \mathrm{~m}$.

A análise laboratorial dos atributos químicos e físicos do solo foi realizada de acordo com o manual proposto pela EMBRAPA (1997) sendo analisados os seguintes atributos químicos do solo: cálcio $(\mathrm{Ca})$ e magnésio $(\mathrm{Mg})$ trocáveis, fósforo $(\mathrm{P})$ e potássio (K) disponível, $\mathrm{pH}$ e carbono orgânico, este na forma de matéria orgânica (MO) e atributos físicos: estabilidade de agregados para determinação do diâmetro médio geométrico com $\left(D \mathrm{DG}_{\mathrm{cp}}\right)$ e sem $\left(\mathrm{DMG}_{\mathrm{sp}}\right)$ pré- 
A.

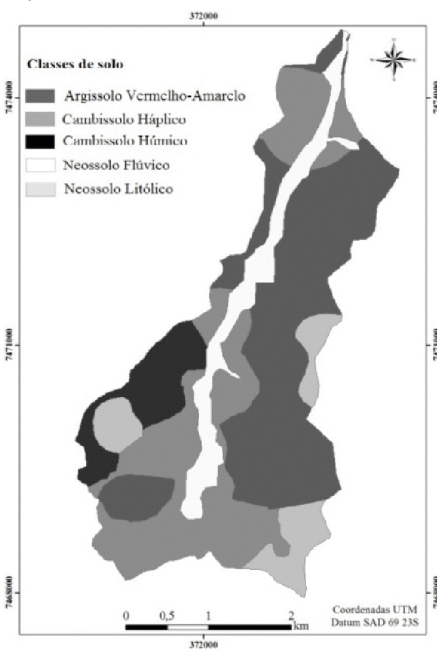

B.

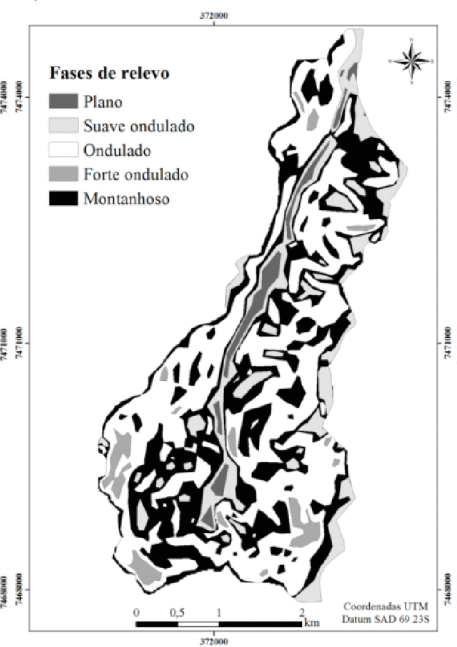

C.

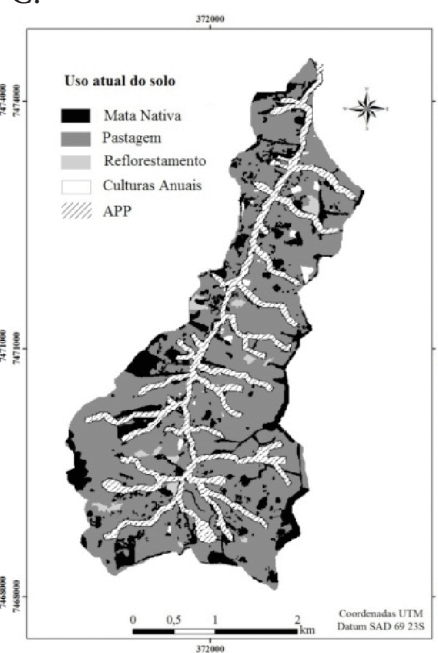

D.

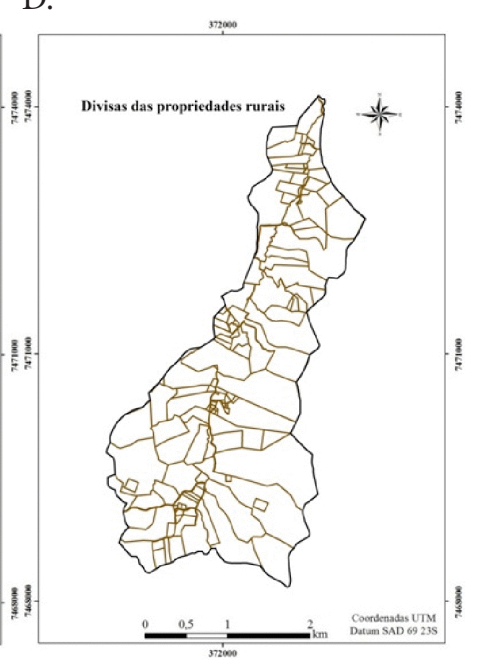

Figura 1. Mapa das classes de solo (A), fases de relevo (B), uso atual do solo (C) e divisa das propriedades (D) da Sub-Bacia das Posses

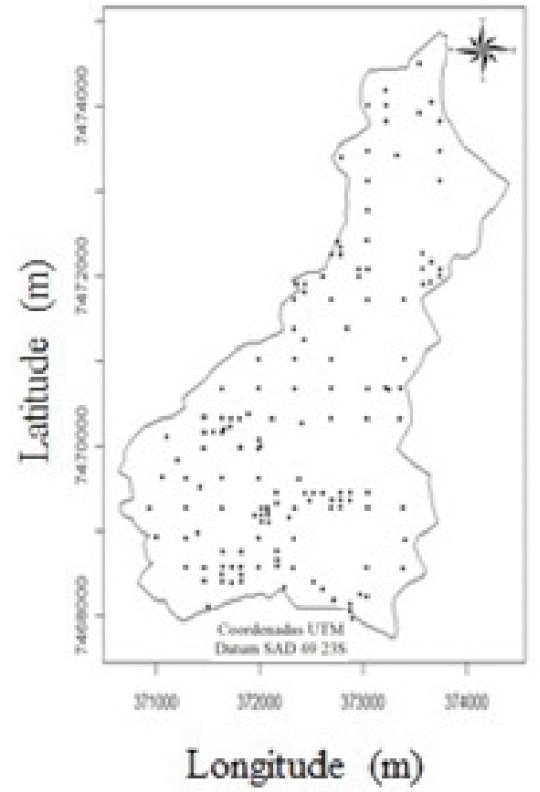

Figura 2. Mapa dos pontos amostrados na Sub-Bacia das Posses

umedecimento e diâmetro médio ponderado com $\left(\mathrm{DMP}_{\mathrm{cp}}\right) \mathrm{e}$ sem $\left(\mathrm{DMP}_{\mathrm{sp}}\right)$ pré-umedecimento, além de argila dispersa em água para determinação do índice de floculação (IF).

A estabilidade de agregados foi determinada através de peneiramento em água com agregados pré-umedecidos lentamente por capilaridade e agregados, os quais não foram submetidos a um pré-umedecimento. A fração de agregados que passou na peneira de 7,93 $\mathrm{mm}$ e ficou retida na peneira de $4,76 \mathrm{~mm}$, foi separada em classes utilizando-se jogo de peneiras de malha: 2, 1, 0,5, 0,25 e 0,105 mm, cujos resultados foram expressos em termos de diâmetro médio geométrico com préumedecimento $\left(\mathrm{DMG}_{\mathrm{cp}}\right)$ e sem pré-umedecimento $\left(\mathrm{DMG}_{\mathrm{sp}}\right)$, diâmetro médio ponderado com pré-umedecimento $\left(\mathrm{DMP}_{\mathrm{cp}}\right)$ e sem pré-umedecimento (DMP ${ }_{\text {sp }}$ ).

Os cálculos dos índices de agregação do solo DMP e DMG, foram realizados para as cinco classes de solo e usos correspondentes da seguinte maneira, aplicando-se as Eqs. 1,2 e 3 .

a) Diâmetro médio geométrico (DMG):

$$
\begin{gathered}
\mathrm{DMG}=10^{\mathrm{X}} \\
\mathrm{X}=\left[\frac{\sum(\mathrm{n} \log \mathrm{d})}{\sum \mathrm{n}}\right]
\end{gathered}
$$

em que:

n - porcentagem dos agregados retidos em determinada peneira

d - diâmetro médio de uma faixa de tamanho do agregado em milímetros

b) Diâmetro médio ponderado (DMP):

$$
\mathrm{DMP}=\sum_{\mathrm{i}=1}^{\mathrm{n}} \mathrm{n}_{\mathrm{i}} \mathrm{d}_{\mathrm{i}}
$$

em que:

$n_{i}$ - porcentagem dos agregados retidos em determinada peneira (forma decimal)

$\mathrm{d}_{\mathrm{i}}$ - diâmetro médio de uma faixa de tamanho do agregado, em milímetros

A argila dispersa em água foi determinada pelo método de Boyoucos. Os valores de argila dispersa em água foram utilizados para cálculo do índice de floculação o qual expressa o grau de dispersão das partículas do solo, sendo importante na agregação do solo (EMBRAPA, 1997).

O cálculo dos índices de floculação do solo foi realizado aplicando-se a Eq. 4.

$$
\mathrm{IF}=\frac{(\mathrm{a}-\mathrm{b})}{\mathrm{a}} * 100
$$


em que:

$$
\begin{array}{ll}
\text { IF } & \text { - índice de floculação em porcentagem } \\
\text { a } & \text { - argila dispersa em } \mathrm{NaOH} \\
\mathrm{b} & \text { - argila dispersa em água }
\end{array}
$$

A análise descritiva dos dados foi obtida em ambiente $R(R$ Development Core Team, 2006), que forneceu os principais momentos estatísticos: média, desvio-padrão, coeficiente de variação e assimetria. A razão de dependência espacial entre amostras foi determinada conforme Cambardella et al. (1994) (Eq. 5):

$$
\mathrm{RD}=\frac{\left(\mathrm{C}_{0}\right)}{\left(\mathrm{C}_{0}+\mathrm{C}_{1}\right)} * 100
$$

em que:

$$
\begin{aligned}
& \mathrm{RD} \text { - razão de dependência espacial } \\
& \mathrm{C}_{0} \text { - efeito pepita } \\
& \left(\mathrm{C}_{0}+\mathrm{C}_{1}\right) \text { - patamar }
\end{aligned}
$$

O estudo da variabilidade espacial foi realizado em ambiente GeoR (R Development Core Team, 2006) com base na análise de semivariogramas em relação às pressuposições da hipótese intrínseca, que diz que a relação de dependência espacial é a mesma em qualquer posição de "h" dentro de determinado alcance da continuidade espacial.

O semivariograma foi estimado pela Eq. 6.

$$
\hat{\gamma}(h)=\frac{\sum_{i=1}^{n(h)}[z(x i)-z(x i+h)]^{2}}{2 n(h)}
$$

em que:

$\mathrm{n}(\mathrm{h})$ - número de pares experimentais de dados separados por uma distância $h$

$\mathrm{z}(\mathrm{xi})$ - valor determinado em cada ponto amostrado

$\mathrm{z}(\mathrm{xi}+\mathrm{h})$ - valor medido em um ponto mais distância $h$

Para cada atributo do solo foram realizados os cálculos das semivariâncias $\gamma(\mathrm{h})$, em todas as direções atendendo à hipótese de isotropia. Após o ajuste de um modelo matemático realizado visualmente ("a sentimento") foram definidos os seguintes parâmetros: efeito pepita $\left(\mathrm{C}_{0}\right)$, valor de $\gamma$ quando h é zero; alcance (a), valor de h quando a semivariância $(\gamma)$ se estabiliza próximo a um valor constante sendo considerado o limite da dependência espacial da grandeza medida; $\left(C_{1}\right)$, variância estrutural e patamar $\left(\mathrm{C}_{0}+\mathrm{C}_{1}\right)$, valor da semivariância $(\gamma)$ quando se obtém um valor constante próximo à variância dos dados (é o máximo da semivariância). O patamar é atingido quando a variância dos dados se torna constante com as distâncias entre as amostras e então este parâmetro permite a determinação da distância limite entre a dependência e a independência entre as amostras. Após a seleção do semivariograma da variável em estudo e havendo dependência espacial pode-se interpolar valores em qualquer posição na área estudada sem tendência e com variância mínima.

De posse dos dados necessários para originar a krigagem, foram construídos os mapas utilizando-se também o GeoR ( $\mathrm{R}$ Development Core Team, 2006).

\section{Resultados e Discussão}

Os resultados referentes à análise descritiva para os valores de $\mathrm{Ca}, \mathrm{Mg}, \mathrm{K}, \mathrm{P}, \mathrm{pH}$, matéria orgânica, $\mathrm{DMG}_{\mathrm{cp}}, \mathrm{DMG}_{\mathrm{sp}}, \mathrm{DMP}_{\mathrm{cp}}$, $\mathrm{DMP}_{\mathrm{sp}}$ e IF são apresentados na Tabela 1.

Os valores de assimetria demonstram distribuições assimétricas para os atributos estudados. O coeficiente de assimetria é mais sensível a valores extremos que a média e desvio padrão, já que um único valor pode influenciar bastante este coeficiente (Isaacs \& Srivastava, 1989). Os valores de assimetria negativa demonstram haver tendência de concentração dos valores acima da média observada sendo mais expressiva tal tendência quanto maior for o valor obtido; para valores positivos deste coeficiente a situação é inversa (Neves Neto et al., 2013).

Nota-se que, apesar de os atributos apresentarem assimetria

\begin{tabular}{|c|c|c|c|c|c|c|}
\hline Variável & Média & Mediana & Desvio padrão & CV (\%) & Classificação do CV 1 & Assimetria \\
\hline $\mathrm{Ca}\left(\mathrm{cmol}_{\mathrm{c}} \mathrm{dm}^{-3}\right)$ & 2,02 & 1,80 & 1,55 & 74,87 & Alta & 0,53 \\
\hline $\mathrm{Mg}\left(\mathrm{cmol}_{\mathrm{c}} \mathrm{dm}^{-3}\right)$ & 0,72 & 0,70 & 0,47 & 63,44 & Alta & 0,26 \\
\hline $\mathrm{K}\left(\mathrm{mg} \mathrm{dm}^{-3}\right)$ & 97,31 & 80 & 63,13 & 65,16 & Alta & 0,80 \\
\hline$P\left(\mathrm{mg} \mathrm{dm}^{-3}\right)$ & 7,04 & 2,60 & 12,12 & 172,51 & Alta & 1,10 \\
\hline MO $\left(\mathrm{g} \mathrm{kg}^{-1}\right)$ & 3,59 & 3,60 & 1,27 & 35,28 & Média & $-0,03$ \\
\hline $\mathrm{pH}$ em $\mathrm{H}_{2} \mathrm{O}$ & 5,44 & 5,50 & 0,37 & 6,85 & Baixa & $-0,42$ \\
\hline $\mathrm{DMG}_{\mathrm{cp}}(\mathrm{mm})$ & 4,03 & 4,21 & 0,40 & 9,84 & Baixa & $-1,34$ \\
\hline $\mathrm{DMG}_{\mathrm{sp}}(\mathrm{mm})$ & 4,02 & 4,29 & 0,97 & 24,21 & Baixa & $-0,81$ \\
\hline $\mathrm{DMP}_{\mathrm{cp}}$ & 4,53 & 4,63 & 0,40 & 8,83 & Baixa & $-0,75$ \\
\hline $\mathrm{DMP}_{\mathrm{sp}}$ & 4,05 & 4,42 & 0,79 & 19,61 & Baixa & $-1,40$ \\
\hline IF (\%) & 30,37 & 30,60 & 8,90 & 29,32 & Baixa & $-0,08$ \\
\hline
\end{tabular}
os dados não apresentam assimetria acentuada de vez que apresentam valores de média e mediana em sua maioria próximos ao banco de dados atendendo às condições de normalidade, ou seja, os valores não são dominados por valores atípicos de distribuição e são mais adequados para uso da geoestatística (Cambardella et al., 1994). Isaacs \& Srivastava

Tabela 1. Parâmetros estatísticos para os atributos químicos e físicos do solo na Sub-Bacia das Posses, Extrema, MG

CV - Coeficiente de variação; Ca - Teor de cálcio; Mg - Teor de magnésio; K - Teor de potássio; P - Teor de fósforo; pH - Potencial hidrogeniônico; M0 - Matéria orgânica do solo; DMG - Diâmetro médio geométrico com pré-umedecimento; $\mathrm{DMG}_{\mathrm{sp}}$ - Diâmetro médio geométrico sem pré-umedecimento; $\mathrm{DMP}_{\mathrm{cp}}$ - Diâmetro médio ponderado com pré-umedecimento; $\mathrm{DMP}_{\mathrm{sp}}$ - Diâmetro médio ponderado sem pré-umedecimento; IF - Índice de floculação; ${ }^{1}$ Classificação segundo Warrick \& Nielsen (1980) 
(1989) destacam que, apesar de importante, a normalidade não é considerada uma exigência para aplicação da geoestatística sendo que a ocorrência de média e variabilidade dos dados constantes é mais importante porque permite que a krigagem não seja comprometida.

Nota-se, a partir da Tabela 1, que a maioria dos atributos químicos avaliados obteve CV superior a $65 \%$, o CV encontrado para o fósforo foi superior a $170 \%$ e apenas o $\mathrm{pH}$ do solo obteve CV baixo, de 6,85\%, o que condiz com uma área mais homogênea para este atributo. Pereira et al. (2013) também verificaram $\mathrm{CV}$ alto para $\mathrm{Ca}, \mathrm{Mg}, \mathrm{P}$ e $\mathrm{K}$ em um Latossolo Vermelho argiloso sob cultivo.

De acordo com a classificação proposta por Warrick \& Nielsen (1980) o coeficiente de variação (CV) indica, quando maior que $35 \%$, uma série heterogênea, $\mathrm{CV}$ maior que $65 \%$, relacionados a um material ainda mais heterogêneo; mesmo assim, média inferior a $35 \%$ representa uma série mais homogênea.

$\mathrm{O}$ alto $\mathrm{CV}$ para atributos químicos pode estar relacionado ao manejo do solo. Na Sub-Bacia das Posses ocorrem diversos usos do solo (Lima, 2010), como: pastagem, mata de eucalipto, culturas anuais como batata e milho e há presença de faixas com mata nativa. Esta diversidade de ambientes condiciona diferenças nas propriedades do solo, em especial dos atributos químicos. Atributos químicos do solo são influenciados pela cultura e práticas agrícolas (Silva \& Lima, 2012) enquanto a adubação foi apontada como a causa dos elevados valores do CV para P e Mg em estudo desenvolvido por Souza et al. (2004).

Os atributos físicos do solo avaliados se comportaram como atributos mais homogêneos em relação ao CV (Warrick \& Nielsen, 1980); ainda assim se notou maior CV para os atributos relacionados à agregação do solo (DMG e DMP) quando estes não foram submetidos a um pré-umedecimento concordando com os resultados encontrados por Oliveira et al. (2013) em um Cambissolo Háplico sob agrofloresta. O pré-umedecimento simula a presença contínua de água nos poros das partículas de solo agindo como a ocorrência de uma precipitação; já a avaliação das amostras do solo sem pré-umedecimento simula o oposto, isto é, condiz com um solo completamente seco em que, quando ocorre uma precipitação que há muito não ocorria, o solo facilmente desagrega podendo causar erosão hídrica. Segundo Andrade \& Rando (1981) a rápida absorção de água pela compressão do ar no interior dos agregados em tratamentos sem pré-umedecimento faz com que a pressão exceda a coesão das partículas ocorrendo a quebra (slaking). Solos que apresentam maior agregação podem ser considerados superiores aos com menor agregação considerando-se a mesma classe de solo (Vezzani \& Mielniczuk 2011).

Os parâmetros de ajuste do semivariograma são apresentados na Tabela 2. O alcance da autocorrelação espacial, ou seja, a distância máxima à qual os atributos estão espacialmente correlacionados (Vieira et al., 1983) foi comparada para os diferentes atributos avaliados. Na Tabela 2 pode-se observar que os valores do alcance obtidos variam de 706,50 a 6.444,00 $\mathrm{m}$, os quais correspondem aos raios das áreas consideradas homogêneas para cada variável estudada. Desta forma, todos os
Tabela 2. Parâmetros de ajuste do semivariograma experimental para os atributos físicos e químicos relacionados com a qualidade do solo em relação à erosão hídrica para a Sub-Bacia das Posses

\begin{tabular}{|c|c|c|c|c|c|c|}
\hline Variável & Modelo & $\mathbf{C}_{0}$ & $C_{1}$ & $C_{0}+C_{1}$ & A & RD \\
\hline $\mathrm{Ca}$ & Wave & 1,62 & 2,12 & 3,74 & 706,50 & 43,31 \\
\hline $\mathrm{Mg}$ & Exponencial & 0,09 & 0,33 & 0,42 & $3.532,00$ & 21,42 \\
\hline K & Exponencial & $2.212,00$ & $4.267,00$ & $6.479,00$ & $3.091,00$ & 34,14 \\
\hline$P$ & \multicolumn{6}{|c|}{ Efeito Pepita Puro } \\
\hline pH & Esférico & 0,03 & 0,11 & 0,14 & 794,50 & 21,42 \\
\hline MO & Exponencial & 1,24 & 1,04 & 2,28 & $3.621,00$ & 54,38 \\
\hline $\mathrm{DMG}_{\mathrm{cp}}$ & Exponencial & 0,18 & 0,45 & 0,63 & $1.128,00$ & 28,57 \\
\hline $\mathrm{DMG}_{\mathrm{SP}}$ & \multicolumn{6}{|c|}{ Efeito Pepita Puro } \\
\hline $\mathrm{DMP}_{\mathrm{cp}}$ & Exponencial & 0,02 & 0,18 & 0,20 & $1.074,00$ & 10,00 \\
\hline $\mathrm{DMP}_{\mathrm{SP}}$ & \multicolumn{6}{|c|}{ Efeito Pepita Puro } \\
\hline IF & Exponencial & 58,61 & 87,91 & 146,52 & $6.444,00$ & 59,99 \\
\hline
\end{tabular}

$C_{0}$ - Efeito pepita; $C_{1}$ - Variância estrutural; $C_{0}+C_{1}-$ Patamar, a - Alcance; RD - Razão de dependência espacial; $M 0$ - Matéria orgânica do solo; $\mathrm{DMG}_{\mathrm{cp}}$ - Diâmetro médio geométrico com pré-umedecimento; $\mathrm{DMG}_{\mathrm{sp}}$ - Diâmetro médio geométrico sem pré-umedecimento; $\mathrm{DMP}_{\mathrm{cD}}$ - Diâmetro médio ponderado com pré-umedecimento; $\mathrm{DMP}_{\mathrm{sp}}$ - Diâmetro médio ponderado sem pré-umedecimento; IF - Índice de floculação

vizinhos situados dentro de um círculo com esses raios, podem ser usados para estimar valores para qualquer ponto entre eles (Vieira \& Lombardi Neto, 1995). Observa-se que os atributos que apresentaram menor e maior alcance foram o teor de $\mathrm{Ca} e$ o índice de floculação (IF) respectivamente.

A partir da razão de dependência espacial (RD) proposta por Cambardella et al. (1994) os atributos avaliados foram classificados como de forte e moderada dependência espacial. Semivariogramas que apresentam razão de dependência (RD) espacial menor ou igual a $25 \%$ têm forte dependência espacial. A dependência é moderada quando esta relação varia de 25 a $75 \%$ e fraca se este valor for superior a $75 \%$ de acordo com esta classificação. A RD moderada, observada para $\mathrm{Ca}, \mathrm{K}, \mathrm{MO}$ e IF se relaciona com os fatores extrínsecos, os quais são promovidos pela ação antrópica, a exemplo da adubação, conforme se inferiu a respeito dos elevados resultados de $\mathrm{CV}$ obtidos nas análises dos atributos químicos. Nenhum dos atributos do solo avaliados apresentou forte dependência espacial, a qual se correlaciona a fatores intrínsecos como clima, relevo e material de origem, segundo Cambardella et al. (1994).

A maioria dos atributos se ajustou ao modelo exponencial, em concordância com Oliveira et al. (1999), Lima et al. (2010) e Neves Neto et al. (2013) que indicaram os modelos exponenciais e esféricos como os mais comuns aos atributos do solo. Para o Ca e pH, os modelos ajustados foram, respectivamente, "wave" e esférico. O P não apresentou dependência espacial sendo representado com efeito pepita puro; o fósforo é um elemento químico essencial ao crescimento das plantas e possui uma característica peculiar quanto à sua necessidade no plantio. Tal elemento é pouco disponível em solos do Brasil e é extremamente reativo; desta maneira, tende a reagir quando aplicado ao solo formando compostos de baixa solubilidade. Ao contrário dos demais nutrientes a adubação com fósforo assume, portanto, a particularidade de se ter que aplicar uma quantidade maior do que aquela exigida pelas plantas haja vista que, primeiro, se torna necessário satisfazer a exigência do solo. 
O manejo do solo, principalmente relacionado à adição de matéria orgânica, é muito importante para a disponibilidade deste nutriente. Em razão do comportamento peculiar do fósforo não foi possível, no presente trabalho, identificar uma dependência espacial do fósforo. A distância máxima de até $350 \mathrm{~m}$ entre cada ponto coletado não foi o suficiente para indicar uma dependência espacial desse nutriente. Este resultado também foi verificado em Souza et al. (1998) que observaram maior variabilidade espacial e ausência de dependência espacial para o fósforo quando avaliado em solo preparado com escarificação.

Os modelos esféricos e exponenciais se apresentam como modelos mais comuns atribuídos ao comportamento do solo e da planta, segundo Cambardella et al. (1994), e Alvarenga et al. (2011). Os atributos químicos têm maior variabilidade espacial devido, possivelmente, à interferência antrópica, como o uso de práticas agrícolas na área (Kavianpoor et al., 2012).

$\mathrm{O}$ ajuste do Ca ao modelo "wave" pode ser explicado pela aplicação de calcário pontualmente na Sub-Bacia das Posses. Na presente sub-bacia vivem aproximadamente 100 proprietários rurais que utilizam a agricultura familiar e pecuária extensiva, como subsistência (Figura 1D). Para tal são plantadas culturas anuais e cultivados pastos (Figura 1C). A sub-bacia é caracterizada por agricultura e pecuária de baixo nível tecnológico porém em algumas áreas os proprietários fazem, durante o preparo do solo, a calagem visando corrigir a elevada acidez do solo. Ao serem aplicadas dosagens de calagem em alguns pontos da sub-bacia, ocorre variação do teor de cálcio ora elevada nesses pontos e ora muito baixa nas demais localidades da Sub-Bacia das Posses, estando o Ca presente em elevado teor, pontualmente, e em menor teor na maior parte da Sub-Bacia, como ocorre com os demais atributos químicos do solo.

Para o $\mathrm{DMG}_{\mathrm{sp}}$ e $\mathrm{DMP}_{\mathrm{sp}}$ não foi possível verificar uma dependência espacial, diferentemente de quando avaliados com pré-umedecimento fato que pode ser explicado pela grande variabilidade dos resultados quando não foi realizado pré-umedecimento antes da análise de estabilidade de agregados. Agregados pré-umedecidos anteriormente à análise, têm comportamento diferente. Quando não é realizado tal procedimento, ou seja, quando estão completamente secos, esses agregados são bastante influenciados ao entrar em contato com água.

Este condicionamento diferente gera grandes variações nos resultados obtidos demostrando uma insuficiência na distância máxima entre pontos utilizados para indicar dependência espacial, originando efeito pepita puro para os atributos $D M G_{\text {sp }}$ e $\mathrm{DMP}_{\text {sp }}$.

Torna-se de extrema importância a presente avaliação tendo em vista que as mesmas variáveis que indicam capacidade de agregação do solo quando analisadas de maneiras diferentes, poderão não indicar dependência espacial (Figura 3, Tabela 2).

Constatou-se, para a maioria dos atributos avaliados, dependência espacial, como mostram os semivariogramas sendo possível, desta maneira, realizar a interpolação de valores em qualquer posição no campo em estudo, elaborando-se os mapas através do processo da krigagem ordinária (Vieira et al., 2011). A krigagem é uma técnica de estimação de locais não amostrados, usando propriedades estruturais do semivariograma confeccionados a partir de locais amostrados. Mapas de krigagem podem ser elaborados para aqueles atributos que apresentam dependência espacial e esta informação é usada para visualizar e melhor entender o padrão de distribuição espacial, além de definir diferentes zonas de manejo em determinada área (Özgöz et al., 2012).

Os mapas de distribuição obtidos por meio da interpolação dos dados, pelo método da krigagem ordinária, são fundamentais na avaliação da agregação do solo de áreas degradadas ou não, sejam potenciais para recarga de água utilizadas na agropecuária ou de preservação permanente. A agregação do solo é influenciada principalmente por atributos químicos e físicos do solo bem como seu manejo e uso. A variabilidade espacial dos atributos físicos e químicos do solo possui relação direta com a manutenção da conservação dos mesmos (Vieira et al., 2011). Esses mapas gerados possibilitam um manejo adequado da área e indicam áreas prioritárias de recuperação, ou seja, aquelas que possuem deficiências nutricionais e impedimentos físicos quanto à agregação do solo que podem ocasionar perdas de solo por erosão hídrica e diminuir a infiltração de água no solo diminuindo também a recarga do lençol freático. A SubBacia das Posses está localizada em área com remanescentes de Mata Atlântica e inserida no Sistema Cantareira, sendo de importância capital a avaliação dos atributos físicos e químicos do solo para conservar e proteger ecossistemas e recuperar áreas que possuem solos com baixo teor de nutrientes e baixos teores de indicadores físicos. Desta forma, são dadas condições a esses solos para a infiltração de água e a possibilidade de crescimento e desenvolvimento de espécies conservando, portanto, uma área de grande importância ecológica, econômica e social (Ribeiro et al., 2009).

Nos mapas gerados pode-se visualizar, na área amostrada, a distribuição espacial dos teores dos nutrientes avaliados, tal como $\mathrm{pH}$, matéria orgânica, diâmetro médio ponderado e índice de floculação. Os mapas de distribuição se mostram mais homogêneos para $\mathrm{Ca}, \mathrm{Mg}$ e $\mathrm{K}$ e apresentam forte dependência espacial (Figura 5 e Tabela 2) indicando baixo teor dos mesmos em grande parte da sub-bacia. Gontijo et al. (2012) verificaram variação no teor de matéria orgânica do solo ocasionada por ação antrópica indicando dependência espacial deste atributo.

Segundo Oliveira et al. (1999) o conhecimento dos valores do alcance e as localizações das áreas em que estão concentrados os maiores e/ou menores valores de determinada variável química, são relevantes para o planejamento do manejo da fertilidade do solo, tanto na agricultura quanto na manutenção da conservação da área. Mesmo a área possuindo baixa fertilidade, é possível, a partir da Figura 5, mostrar as subáreas mais críticas em relação a esses baixos teores. Este fato pode indicar as áreas prioritárias de conservação, ou seja, aquelas mais pobres quanto à presença de nutrientes e matéria orgânica. 

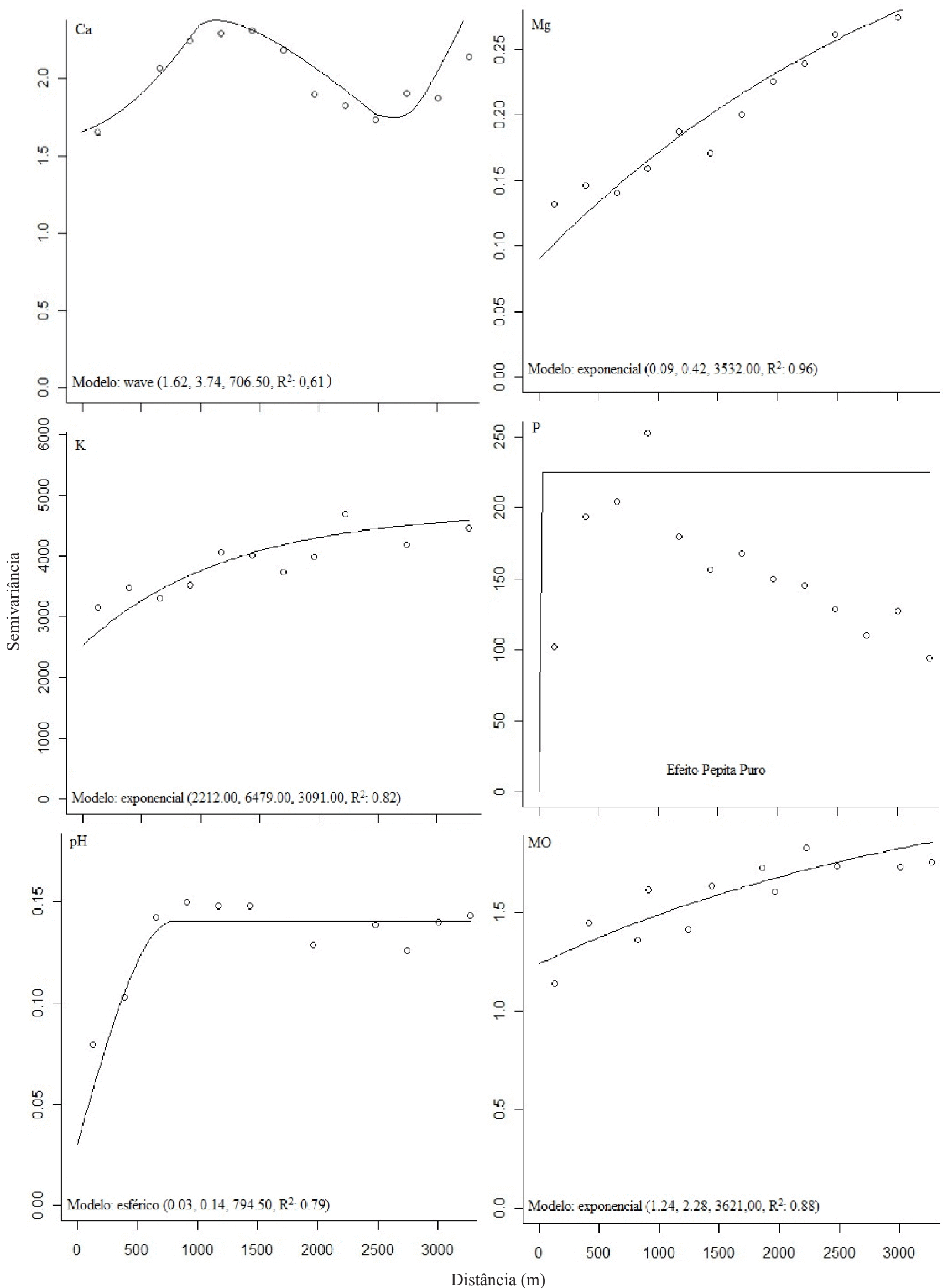

Figura 3. Semivariogramas dos atributos químicos do solo: cálcio, magnésio, potássio, fósforo, pH e matéria orgânica do solo na Sub-Bacia das Posses, Extrema, MG 


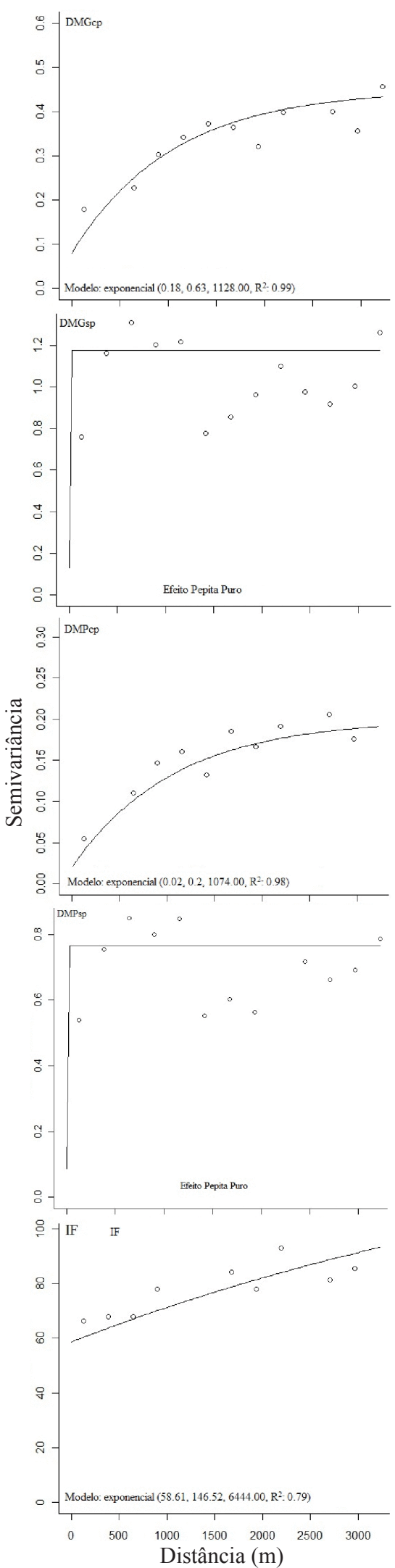

Figura 4. Semivariogramas dos atributos físicos do solo: diâmetro médio geométrico com e sem préumedecimento ( $\mathrm{DMG}_{\mathrm{cp}}$ e $\mathrm{DMG}_{\mathrm{sp}}$ ), diâmetro médio ponderado com e sem pré-umedecimento DMP $_{c p}$ e DMP ${ }_{s p}$, índice de floculação (IF) na Sub-Bacia das Posses, Extrema, MG

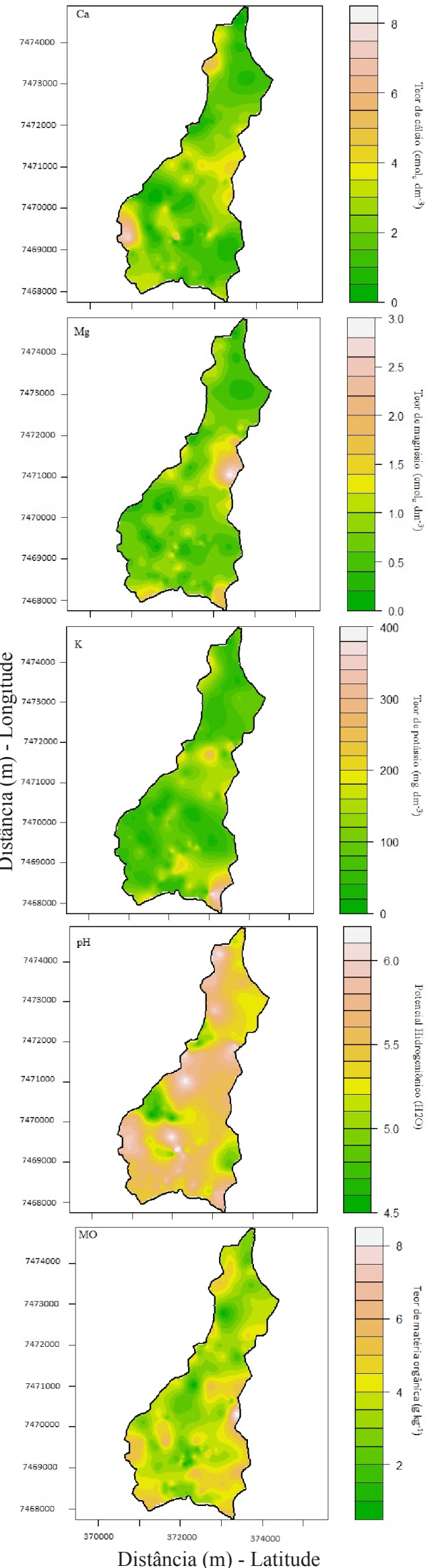

Figura 5.Mapa de distribuição dos atributos químicos do solo na Sub-Bacia das Posses, Extrema, MG 


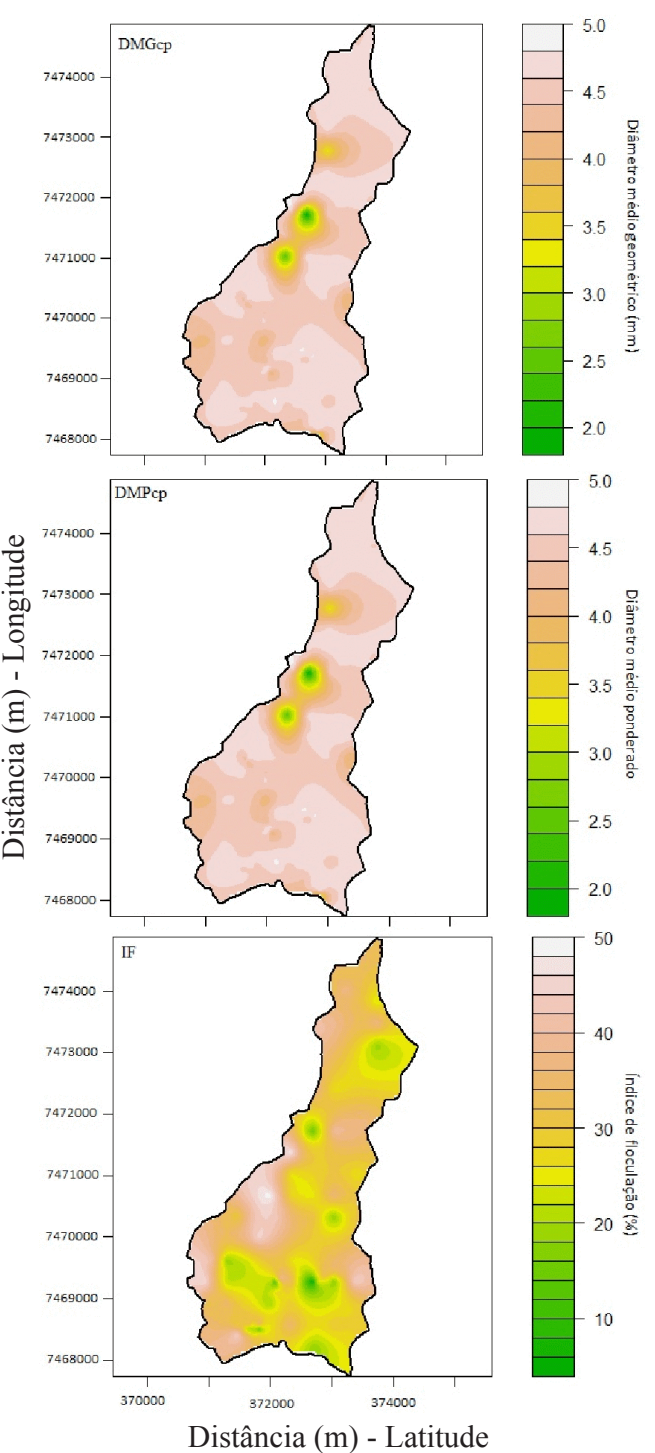

Figura 6. Mapa de distribuição dos atributos físicos do solo na Sub-Bacia das Posses, Extrema (MG)

Observa-se, nos mapas de distribuição (Figuras 5 e 6) que ao norte da área, onde é encontrado relevo montanhoso, predominam a classe de solo Cambissolo (Figura 1A) e a pastagem (Figura 1C) ocorrem menores valores de cálcio, magnésio, potássio e, consequentemente, uma acidez maior evidenciada por menores valores de $\mathrm{pH}$, além de teores medianos de matéria orgânica e de índice de floculação e elevados teores de diâmetro médio ponderado. Deste modo, o complexo de troca é preferencialmente ocupado por cátions de caráter ácido uma vez que os cátions de caráter básico podem ser removidos pela água da chuva para locais mais baixos do terreno, corroborando com as observações de Souza et al. (2008) para cálcio e saturação de bases em áreas com pastagem.

\section{Conclusões}

1. As variáveis estudadas apresentaram variabilidade espacial, de acordo com a posição em que se encontram na paisagem. Esta estrutura de dependência espacial variou de forte a moderada e possibilitou o mapeamento através de ferramentas da geoestatística com exceção do P, DMG e DMP avaliados sem pré-umedecimento.

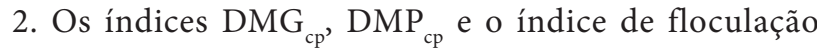
avaliados foram ajustados em conformidade entre si, na maior parte da Sub-Bacia.

3. Os índices físicos avaliados indicaram boa agregação do solo.

4. A variabilidade natural dos atributos químicos do solo associada à aplicação e incorporação de calcário em pequenas áreas isoladas acarretou, de maneira geral, em baixos teores de nutrientes nos solos da Sub-Bacia com altas concentrações de nutrientes em alguns pontos difusos na bacia.

\section{Agradecimentos}

À CAPES e ao CNPq, pela concessão da bolsa aos autores e coautores deste trabalho; à FAPEMIG, pelo financiamento de parte do projeto (CAG-APQ-01423-11 e CAG-PPM-00422-11); ao CNPq, pelo financiamento do projeto vigente no 471522/2012 e à Prefeitura Municipal de Extrema, Minas Gerais, na pessoa do Secretário de Meio Ambiente, Paulo Henrique Pereira, pelo suporte logístico e ao funcionário, Benedito Arlindo Cortes, pelo auxílio nos trabalhos de campo.

\section{Literatura Citada}

Alvarenga, C. C.; Mello, C. R.; Mello, J. M.; Viola, M. R. Continuidade espacial da condutividade hidráulica saturada do solo na bacia hidrográfica do Alto Rio Grande, MG. Revista Brasileira de Ciência do Solo, v.35, p.1745-1758, 2011.

ANA - Agência Nacional de Águas. Programa produtor de água superintendência de usos múltiplos. Brasília: Ministério do Meio Ambiente, 2008.

Andrade, J. C. F. H.; Rando, E. M. Alterações na consistência e textura de um latosolo do roxo distrófico ocasionado pelo cultivo convencional. Revista Ciência e Prática, v.5, p.4047, 1981.

Cambardella, C. A.; Moorman, T. B.; Novak, J. M.; Parkin, T. B.; Karlen, D. L.; Turco, R. F. \& Konopka, A. E. Field-scale variability of soil properties in Central Iowa Soils. Soil Science Society of America Journal, v.58, p.1501-1511, 1994.

EMBRAPA - Empresa Brasileira de Pesquisa Agropecuária. Manual de métodos de análise de solo. 2.ed. Rio de Janeiro: Ministério da Agricultura, 1997. 212p.

Grego, C. R.; Vieira, S. R. Variabilidade espacial de propriedades físicas do solo em uma parcela experimental. Revista Brasileira de Ciência do Solo, v.29, p.169-177, 2005.

Gontijo, I.; Nicole, L. R.; Partelli, F. L.; Bonomo, R.; Santos; E. O. J. Variabilidade e correlação espacial de micronutrientes e matéria orgânica do solo com a produtividade da pimentado-reino. Revista Brasileira de Ciência do Solo, v.36, p.10931102, 2012. 
Hurtado, S. M. C.; Silva, C. A.; Resende, A. V.; Pinho, R. G. von; Inácio, E. S. B.; Higashikawa, F. S. Spatial variability of soil acidity attribute sand the spatializationoflimingrequirement for corn. Revista Ciência e Agrotecnologia, v.33, p.13511359, 2010.

Isaaks, E. H.; Srivastava, R. M. An introduction to applied geoestatistics. 1.ed. New York: Oxford University Press, 1989. 5p.

Kavianpoor, H.; Esmali Ouri, A.; Jafarian Jeloudar, Z.; Kavian, A. Spatial variability of some chemical and physical soil properties in Nesho Mountainous Rangelands. American Journalof Environmental Engineering, v.2, p.34-44, 2012.

Lima, G. C. Avaliação de atributos indicadores da qualidade do solo em relação à recarga de água na Sub-Bacia das Posses, Extrema (MG). Lavras: UFLA, 2010. 99p. Dissertação Mestrado

Lima, J. S. S.; Souza, G. S.; Silva, S. A. Amostragem e variabilidade espacial de atributos químicos do solo em área de vegetação natural em regeneração. Revista Árvore, v.34, p.127-136, 2010.

MMA - Ministério do Meio Ambiente. Relatório de inspeção: Área atingida pela tragédia das chuvas região serrana do Rio de Janeiro. Brasília: MMA, 2011. 85p.

Neves Neto, D. N.; Santos, A. C. dos; Santos, P. M.; Melo, J. C.; Santos, J. S. Análise espacial de atributos do solo e cobertura vegetal em diferentes condições de pastagem. Revista Brasileira de Engenharia Agrícola e Ambiental, v.17, p.995-1004, 2013.

Oliveira, I. A.; Campos, M. C. C.; Soares, M. D. R.; Aquino, R. E.; Marques Júnior, J.; Nascimento, E. P. do. Variabilidade espacial de atributos físicos em um cambissolo háplico, sob diferentes usos na região sul do Amazonas. Revista Brasileira de Ciência do Solo, v.37, p.1103-1112, 2013.

Oliveira, J. J.; Chaves, L. H. G.; Queiroz, J. E.; Luna, J. G. Variabilidade espacial de propriedades químicas em um solo salino-sódico. Revista Brasileira de Ciência do Solo, v.23, p.783-789, 1999.

Özgöz, E.; Günal, H.; Önen, H.; Bayram, M.; Acir, N. Effect of management on spatial and temporal distribution of soil physical properties. Journal of Agricultural Sciences, v.18, p.70-91, 2012.

Pereira, G. T.; Souza, Z. M. de; Teixeira, D. B.; Montanari, R.; Marques Júnior, J. Optimization of the sampling scheme for maps of physical and chemical properties estimated by kriging. Revista Brasileira de Ciência do Solo, v.37, p.11281135, 2013.
R Development Core Team. R: A language and environment for statistical computing. Vienna: R Foundation for Statistical Computing, 2006. 1731p.

Ribeiro, M. C.; Metzger, J. P.; Martensen, A. C.; Ponzoni, F. J.; Hirota, M. M. The Brazilian Atlantic Forest: How much is left, and how is the remaining forest distributed? Implications for conservation. Biological Conservation, v.142, p.1141-1153, 2009.

Silva, S. A.; Lima, J. S. S. Multivariate analysis and geostatistics of the fertility of a humicrhodichapludox under coffee cultivation. Revista Brasileira de Ciência do Solo, v.36, p.467-474, 2012.

Souza, G. S.; Lima, J. S. S.; Xavier, A. C.; Rocha, W. S. D. Krigagem ordinária e inverso do quadrado da distância aplicados na espacialização de atributos químicos de um argissolo. Revista Scientia Agraria, v.11, p.73-81, 2010.

Souza, G. S. de; Lima, J. S. S.; Silva, S. A.; Oliveira, R. B. de. Variabilidade espacial de atributos químicos em um Argissolo sob pastagem. Acta Scientiarum Agronomy, v.30, p.589-596, 2008.

Souza, L. S.; Cogo, N. P.; Vieira, S. R. Variabilidade de fósforo, potássio e matéria orgânica no solo em relação a sistemas de manejo. Revista Brasileira de Ciência do Solo, v.22, p.7786, 1998.

Souza, Z. M.; Marques Júnior, J.; Pereira, G. T.; Moreira, L. F. Variabilidade espacial do $\mathrm{pH}, \mathrm{Ca}, \mathrm{Mg}, \mathrm{V} \%$, do solo em diferentes formas do relevo sob cultivo de cana-de-açúcar. Revista Ciência Rural, v.34, p.1763-1771, 2004.

Vezzani, F. M.; Mielniczuk, J. Agregação e estoque de carbono em argissolo submetido a diferentes práticas de manejo agrícola. Revista Brasileira de Ciência do Solo, v.35, p.213223, 2011.

Vieira, S. R.; Dechen, S. C. F.; Siqueira, G. M.; Dufranc, G. Variabilidade espacial de atributos físicos e químicos relacionados com o estado de agregação de dois latossolos cultivados no sistema de semeadura direta. Bragantia, v.70, p.185-195, 2011.

Vieira, S. R.; Hatfield, J. L.; Nielsen, D. R.; Biggar, J. W. Geostatistical theory and application to variability of some agronomical properties. Berkeley: University of California, v.51, 1983. 75p.

Vieira, S. R.; Lombardi Neto, F. Variabilidade espacial do potencial de erosão das chuvas do estado de São Paulo. Bragantia, v.54, p.405-412, 1995.

Warrick, A. W.; Nielsen, D. R. Spatial variability of soil physical proprieties in the field. In: Hillel, D. Aplications of soil physics. New York: Academis Press, 1980. Cap.5, p.319-344. 